Int. J. Dev. Biol. 64: 29-34 (2020)

https://doi.org/10.1387/ijdb.190162dk

\title{
Fungal senescence induced by the Neurospora sen mutation and mitochondrial plasmids - the contributions of Ramesh Maheshwari
}

\author{
DURGADAS P. KASBEKAR* \\ Centre for DNA Fingerprinting and Diagnostics, Uppal, Hyderabad, India
}

\begin{abstract}
This article describes some of the research contributions made by Prof. Ramesh Maheshwari and his colleagues at the Indian Institute of Science, Bangalore. These include (1) the understanding of the Neurospora life cycle in agricultural (sugarcane) fields, (2) identification of Neurospora mutants that trigger vegetative spore development via microcycle conidiation, and (3) isolation of wild Neurospora strains in which the essential immortality of the fungal mycelia is subverted.
\end{abstract}

KEY WORDS: furfural, mitochondrial plasmid, nucleus-mitochondria interaction, somatic mutation, syntrophic growth

\section{Introduction}

Senescence is a durable cell-cycle arrest of previously replication-competent cells, most commonly associated with organismal aging, but it is becoming increasingly apparent that the underlying mechanisms are important for "normal" developmental processes too, for instance in morphogenesis and repair of tissue damage ( $\mathrm{He}$ and Sharpless, 2017). The discovery and study of senescence of the multinucleated mycelium of the fungus Neurospora, driven by the nuclear senescent (sen) mutation, or by mitochondrial plasmids, marked the culmination of a part of the research pursued by Prof. Ramesh Maheshwari (Fig. 1) and his colleagues at the Indian Institute of Science, Bengaluru. The research interests of this group included: (1) The understanding of the fungal life cycle in agricultural (sugarcane) fields, (2) identifying mutations that trigger vegetative spore development via microcycle conidiation, and (3) isolating wild strains in which the essential immortality of fungal mycelia is subverted. As far as we know Neurospora nuclei are capable of undergoing unlimited rounds of mitotic divisions, and the resulting daughter nuclei populate the newly extended hyphae or are budded off into vegetative spores called macroconidia and microconidia. This article will briefly describe their contributions in these three areas, and conclude with some perspectives for future research. A brief biographical sketch of Prof. Maheshwari is given at the end of this paper.

\section{Life-cycle of Neurospora in sugarcane fields}

After sugarcane is harvested the residual dry leafy trash is burned, presumably to clear the field in preparation for ratooning. Ratooning is the practice of harvesting the above-ground portion of a monocot crop but leaving the roots and attached shoot apices intact to generate the next season's fresh crop. Pandit and Maheshwari (1996) found that Neurospora grew on the burned stubble in sugarcane fields in Maddur (better known for its savory vade), near Bangalore (now Bengaluru). Using an albino mutant as a marked control in experiments that reconstructed field conditions in the laboratory, they could conclude that Neurospora ascospores are disseminated into the soil rather than into the wind. The ascospores are activated in the soil by furfural released from the burnt cane stumps, and the emerging progeny hyphae first infect the lower portions of the scorched stumps. The mycelia grow into the sugarrich tissues and produce copious amounts of mycelial mats and macroconidia that are either dispersed into the air or foraged upon by the microfauna such as mites and nematodes. The constant production and dispersal of macroconidia and mycelia, together with the foraging by the microfauna, mechanically ruptures the epidermis of the sugarcane and drains the plant tissue of sugar

Abbreviations used in this paper: mat, mating type; sen, senescent.

\footnotetext{
*Address correspondence to: Durgadas P. Kasbekar. Centre for DNA Fingerprinting and Diagnostics, Uppal, Hyderabad 500039, India. E-mail: kas@cdfd.org.in (D) https://orcid.org/0000-0002-5269-8020
} 
and minerals. This creates nutritional conditions favorable for the development of microconidia and protoperithecia, which are the incipient male and female fungal sexual structures. The microconidia are uninucleate and smaller $(2.5-3.5 \mu \mathrm{m})$ than the multinucleate macroconidia (4-7 $\mu \mathrm{m})$, and there is a clear temporal separation in their production in the field; the macroconidia develop first, and the microconidia and protoperithecia develop only after several weeks. The fertilization of protoperithecia by microconidia of the opposite mating type induces their development into mature perithecia, and the progeny ascospores that develop in the perithecia are expelled through its ostiole and carried by rain or irrigation water into the soil. So far as I remember, no one else had proposed that the primary role of macroconidia production was to prepare the stumps for the ensuing sexual cycle, because in the laboratory the macroconida and hyphal fragments also can serve as the male gametes to fertilize the protoperithecia. Additionally, Pandit and Maheshwari suggested that although the thermal-tolerance of ascospores enables them to withstand the high temperatures associated with stubble burning, it is the furfural leached from the burning stumps rather than the heat-treatment that is the primary trigger for ascospore activation. Further, Maheshwari noted that since an ascospore is a product of meiosis, a Neurospora colony formed on sugarcane is expected to be homokaryotic and contain genetically identical mitotically-produced nuclei. However, they found phenotypic variation in cultures derived from microconidia from wild-isolated Neurospora (see below), which indicated that the vegetative mycelia can accumulate "somatic" mutations. These insights into the life-history of Neurospora traits in its natural habitat are novel, as is the attribution of differing physiological roles of the two conidial types. These features of the natural phenomena were apparently overlooked by researchers who relied solely on laboratory-based studies, because the temporal separation of macro- and microconidial development is obscured during

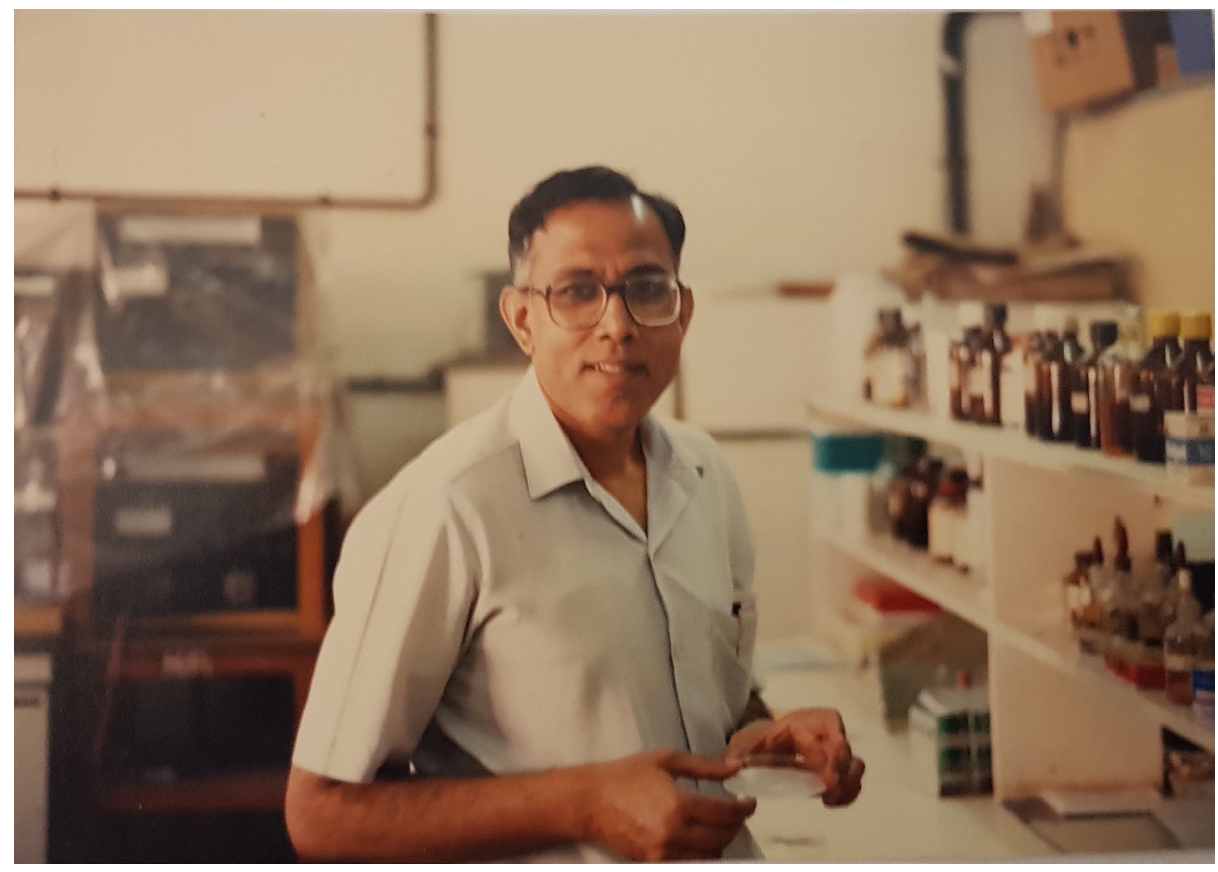

Fig. 1. Ramesh Maheshwari in his laboratory, probably in the mid-1980s. Photo courtesy of Govind Maheshwari. the routine handling of the fungus in the laboratory. Pandit and Maheshwari dedicated the paper to Alfred Sussman and David D Perkins, two stalwarts in Neurospora Genetics who nurtured generations of young researchers in this field.

\section{Microcycle conidiation mutations obtained from wild-isolated $\mathbf{N}$. crassa}

Wild-isolated Neurospora strains can harbor mutations with interesting and unexpected phenotypes, and once the mutations are detected they can be analyzed using the armamentarium of genetic resources available in Neurospora. The isolation by Perkins (1970) of Neurospora from burnt substrates such as sugarcane stubble, corn cobs, and bagasse heaps dumped outside sugar factories inspired Maheshwari and Antony (1974) and Palanivelu and Maheshwari (1979) to look for Neurospora ascospores in soils from all over India. They even enlisted Romulus Whitaker, India's well-known "snake man", to send soil samples from Port Blair, Andaman Islands, from which they isolated an N. sitophilus strain. One wild $N$. crassa strain, Vickramam, from Thanjavur District, Tamil Nadu, showed an unusual developmental phenotype called microcycle conidiation. Microcycle conidiation is seen when macroconidia harvested from agar-grown cultures are germinated in submerged liquid culture where they soon arrest their germ tube elongation, and the germ tube differentiates into macroconidia or microconidia without the typical intervening mycelial phase (Maheshwari, 1989).

In the year 1989, Maheshwari carried out research in Perkins's laboratory at Stanford University on a Fulbright fellowship to genetically analyze the Vickramam strain by crossing it with the strain OR $a$ of the standard laboratory Oak Ridge background. The OR a strain has the opposite mating type (mat a) to Vickramam $A$ ( $m a t$ A). About $30 \%$ of the progeny from the cross showed microcycle conidiation, but the phenotype of many progeny strains differed from that of the Vickramam parent (Maheshwari, 1991). Of the mutant progeny a majority (35/45) produced only macroconidia following microcycle conidiation, whereas the rest (10/45) produced microconidia at $22{ }^{\circ} \mathrm{C}$ but macroconidia at $30^{\circ} \mathrm{C}$. To explain these results Maheshwari (1991) hypothesized that the Vickramam strain contains two mutations, $\mathrm{mcb}$ and $\mathrm{mcm}$, and that $m c b$ was epistatic to $\mathrm{mcm}$. Both the mutations were introgressed into the OR background, and he verified that the $m c b$, and $\mathrm{mcb}$; $\mathrm{mcm}$, progeny produced macroconidia regardless of the temperature, while the $\mathrm{mcm}$ progeny produced microconidia at $22^{\circ} \mathrm{C}$ and macroconidia at $30^{\circ} \mathrm{C}$.

Further, he mapped mcbto linkage group VR, about 3.2 units from al-3, and $\mathrm{mcm}$ to IIL, about 1.4 units from ro-7. By analyzing progeny from an $\mathrm{mcb} \times \mathrm{mcm}$ cross he confirmed the prediction that $m c b$ was epistatic to $\mathrm{mcm}$ in the $\mathrm{mcb}$; $\mathrm{mcm}$ double mutants. Maheshwari hoped that the $\mathrm{mcb}$ and $\mathrm{mcm}$ strains would find use in studies of developmental gene regulation by enabling the 
synchronous production of large quantities of the desired conidial types in a short time (12-24 h). However, the studies he might have had in mind were not subsequently performed by anyone.

\section{A "simple and highly effective method of obtaining pure microconidia" in Neurospora}

Since microconidia are uninucleate they provide an effective method of isolating individual nuclei from the nuclear population in the mycelium. Toward this end, Pandit and Maheshwari (1993) developed a method to obtain them that did not depend on the mcm mutation. A circular piece of cellophane was used to cover the surface of $2 \%$ water agar in a Petri dish, and a culture was initiated at the center of the cellophane by piercing it at the point of inoculation with a trace quantity of macroconidia. A thin mycelial growth developed over the cellophane and produced patches of aerial microconidiophores in 7-12 days. After 10 days of mycelial growth the cellophane was peeled off, and microconidia developed from the thin mycelium which had grown in the agar under the cellophane, and could be harvested in 1-2 ml sterile water. Additional harvests of microconidia could be made after harvesting the previous crop without any significant contamination by macroconidia. [My friend Prof. T. S. Suryanarayanan of Chennai alerted me to his own work demonstrating the production of microconidia via microcycle conidiation on cellophane in other fungi (Suryanarayanan and Rathinakumar, 1984).]

Since Maheshwari had found a way to obtain pure microconidia, it made sense for him to review the literature on microconidiogenesis in Neurospora crassa (Maheshwari, 1999). He noted that although in nature microconidia function as spermatia (male gametes), "for most biologists who use Neurospora, the microconidia have remained unseen, superfluous structures", and he even cited Dodge's (1936) lament that "few persons who have studied species of Neurospora even intensively have ever seen their spermatia". Indeed, for more than 25 years of research in Neurospora Genetics, neither I nor my students have had to use microconidia. Neurospora microconidia are a niche interest, and Maheshwari's 1999 review article helped "regular" Neurospora geneticists like me to "update my ignorance" about them.

\section{Discovery of the senescent (sen) mutant}

Maheshwari et al.,(1994) noticed that senescence was common in $N$. intermedia cultures isolated from sugarcane fields. Of 150 cultures examined by his group, 29 could not be propagated beyond 57 serial subcultures made at intervals of 5-10 days on Vogel's $N$ medium (+ $1.5 \%$ sucrose), and another 30 either lost their vigor or had become aconidial. The senescence began abruptly after about 9 subcultures. A. Navaraj, A. Pandit, and R. Maheshwari (2000) studied one culture in detail by germinating microconidia obtained using the "cellophane" method (see above). Of 159 colonies examined, one showed a progressively weakening growth that ended with mycelial death in 5 to 12 subcultures. The culture was designated Maddur 1991-101-10, named after the place and year of collection-number of original isolate-number of microconidial derivative. In contrast, the mycelium from which the microconidia were derived continued to show normal growth through several passages. Additionally, when the original wild-isolated culture was crossed to the $N$. intermedia standard laboratory strain, 21 of 40 progeny tested showed the senescent phenotype, suggesting that the phenotype segregated as a single recessive nuclear mutation, which was designated senescent (sen). All the progeny from a sen-homozygous cross showed sudden loss of conidiation after a few subcultures and by the 11th subculture all were dead. In contrast, a strain that has not senesced in some 50 subcultures is commonly regarded as immortal, although a wild-type strain was reported to senesce after $12,000 \mathrm{~h}$ of growth, implying that some strains might undergo a late senescence (Kothe et al., 2010). The sen mutation, introgressed into $N$. crassa to facilitate mapping, was mapped in linkage group VR to 4 map units to the right of his-1 and 13 map units to the left of al-3. A sen culture initiated at $26^{\circ} \mathrm{C}$ starts out showing the wild-type growth phenotype, but dies rather abruptly after five to seven passages. On the other hand, when it is initiated at $34^{\circ} \mathrm{C}$, it shows a weak growth right from the beginning. A mutant with this phenotype would have been difficult to produce in the laboratory, therefore its isolation attested to the value of collection and analysis of strains from their natural habitat.

All previously isolated senescent Neurospora strains showed mitochondrial defects. Senescence in the kalilo and the maranhar strains, respectively, from Hawail and Maharashtra, was caused by the presence of linear plasmids in the mitochondria which induced insertional mutagenesis in the mitochondrial genome (Bertrand et al., 1985). The kalilo and the maranharstrain plasmids (respectively, 8642 bp and 7052 bp long) do not share sequence homology. The $N$. crassa natural death (nd) nuclear gene mutant showed high frequencies of deletions and duplications in mtDNA (Seidel-Rogol et al., 1989), suggesting that the $n d^{+}$allele encodes a protein which controls recombination, repair, or replication of mtDNA (Bertrand et al., 1993). This prompted Navaraj et al. (2000) to examine the sen mutant for mitochondrial defects, and they found growth temperature-dependent changes in respiratory chain cytochrome composition. Respiration in wild-type conidia was inhibited by cyanide, indicating that it was predominantly due to the cytochrome chain. In contrast, cyanide had no effect on respiration of sen conidia, indicating that respiration in these strains was via an alternative cyanide-resistant pathway of respiration. Salicyl hydroxamic acid, a specific inhibitor of alternative oxidase, inhibited respiration of sen conidia. The alternative pathway of respiration was much more active in the sen mutant compared to sen ${ }^{+}$during progression of senescence at $26^{\circ} \mathrm{C}$, indicating a shift in electron transport from the cytochrome to the alternate respiratory pathway.

\section{The $\operatorname{sen}^{+}$gene maintains mitochondrial genome integrity}

A. D'Souza, a PhD student of Maheshwari, asked whether the sen mutation causes instability of the mtDNA that then progresses into mitochondrial dysfunction which ultimately results in mycelial death. In collaboration with $\mathrm{H}$. Bertrand (Michigan State University, USA),

D'Souza and Maheshwari performed restriction fragment analysis of mitochondrial genome from senescing mycelia. They cloned and sequenced three novel EcoRI fragments that accumulated in the mtDNA of senescing mycelia. The findings led them to suggest that the fragments resulted from intramolecular recombination between short repeats of 6-10 bp, located close to potential hairpin structures that might facilitate homologous recombination, and their formation also gave rise to deletions in the mitochondrial genome. Presumably, the sen ${ }^{+}$gene product protects the mitochondrial genome from undergoing cruciform-associated recombination 
and deletions. In this respect, its function was similar to that of $n d^{+}$ which suppresses homologous recombination in the mitochondrial genome, and both genes serve to safeguard the mitochondrial genome integrity.

Additionally, D'Souza et al., (2005) found that at least some of the abnormal molecules produced by the cruciform-associated recombination and deletion were suppressive, that is, they displaced the normal mtDNA molecules from the growing mycelia. Thus, the rapid-senescence phenotype likely reflected both generation of mtDNA deletions, as well as the ability of one or several of them to replace the residual normal mtDNA. Notably, [sen $\left.+s e n^{+}\right]$ heterokaryons (the sen+nucleus is the wild type) made by mixing pre-germinated senconidia with ungerminated wild-type conidia also showed mycelial death. Presumably, the suppressive mitochondrial genome forms accumulated by the sen mitochrondria prior to the fusion killed the post-fusion mycelium, despite the subsequent introduction of the dominant sen allele. In contrast, death did not occur when the heterokaryons were constructed by mixing both sen and sen $^{+}$conidia, presumably because the pre-fusion sen cytoplasm had not yet formed the suppressive deletion derivatives of mtDNA, and the recessive sen nuclei could be indefinitely sheltered in the nonsenescent $\left[\right.$ sen + sen $\left.^{+}\right]$heterokaryons made by the latter method. Interestingly, 10 of 12 genotypically al-2 nic-1 sen $^{+}$a homokaryons re-extracted on nicotinamide-supplemented medium from a [(al-1 pan-1 sen a) + (al-2 nic-1 sen s $\left.^{+}\right]$heterokaryon made by the first method showed senescence after subculture on nicotinamide-medium and the other two survived up to four subcultures. Thus, the suppressive mtDNA derivative acted as a transmissible cytoplasmic senescence-causing factor in a heterokaryon formed between the al-1 pan-1 sen a germlings and non-mutant al-2 nic-1 sen+ a conidia.

\section{Plasmid-mediated senescence in the Maddur strains}

Only 12 of the roughly $150 \mathrm{~N}$. intermedia cultures collected from Maddur and stored at $20^{\circ} \mathrm{C}$ in 1991 could be revived after 10 years. In contrast, similarly stored stocks of the $N$. intermedia and

\section{Box 1}

Our unsuccessful strategy to use $f m f-1$ to enrich for RIP-defective mutants

Our strategy was based on the expectation that "closely linked duplicated genome segments almost never escape RIP" (Irelan and Selker, 1996). Identification of the fmf-1 gene enabled the creation of a closely linked duplication via targeted insertion of transforming DNA $~ 5 \mathrm{~kb}$ upstream of the endogenous gene's start codon. We expected that the progeny from the duplication-homozygous crosses would include an overwhelming number that suffered RIPinduced mutations in $f m f-1$, which renders them infertile as either the male or female parent in crosses. Consequently, if the $f 1$ progeny were germinated en masse and allowed to randomly mate, then only the rare non-RIPed progeny would undergo crosses to produce the f2 generation. Likewise, crosses among the $f 2, f 3$, etc, generations would progressively enrich for non-RIPed progeny, and thus enrich for RIP-defective mutants. The strategy was unsuccessful because "only" 85\% of the $f 1$ progeny had suffered RIP-induced mutations in fmf-1 (unpublished results of my PhD student Srividhya lyer). Although impressive, 85\% fell far short of the hoped for 98\%.
N. crassastandard wild-type strains Shp1 aand 74-OR23-1 A were readily revivable. A. D'Souza, S. Sultana and Maheshwari (2005) found that these remaining 12 strains also senesced on Vogel's medium, although their longevity varied from 12 to 70 subcultures. Due to the ease of studying senescence in a strain that died in fewer (12-16) subcultures, they chose the M1991-60A culture for detailed study. They performed the reciprocal crosses M199160 $A$ (female) x Shp1 a (male) and M1991-60 A (male) x Shp1 a (female), and found that all progeny from the cross in which M1991-60 $A$ was the female parent senesced, whereas progeny from the cross in which the Shp1 a strain was the female did not senesce, indicating that senescence was a cytoplasmically inherited trait. The senescing cultures showed mitochondrial dysfunction, and EcoRI restriction digests of their $\mathrm{mtDNA}$ revealed novel 21,6 , and $2.7 \mathrm{~kb}$ fragments, suggesting the occurrence of senescence-associated DNA rearrangements. The cloning and sequencing of novel fragments revealed the presence of a circular $3614 \mathrm{bp}$ mitochondrial retroplasmid designated as pMADDUR1 (pMAD1), which was $>97 \%$ homologous to the retroplasmids pVARKUD (3675 bp) and pMAURICEVILLE (3581 bp) that were previously shown to cause senescence in the $N$. intermedia Varkud strain (India) and the $N$. crassa Mauriceville strain (USA). The pMADDUR1 plasmid was absent from the Shp1 a strain but it was inherited by all the progeny from the M1991-60 A (female) x Shp1 a (male) cross, and in none of the progeny from the reciprocal M1991-60 A (male) $x$ Shp1 a (female) cross. Further, six of the 12 revived strains from the 1991 Maddur collection contained pMAD1, and one strain contained a closely related 3774 bp variant called pMAD2.

Other researchers had shown that the Varkud group of retroplasmids integrate into the mitochondrial genome by first forming variant plasmids that are fusion cDNAs made via reverse transcription of the full length plasmid transcript that is primed by mitochondrial tRNA (Chiang et al., 1994). The tRNA-like structure acts as a recognition sequence for the retroplasmidencoded reverse transcriptase, somewhat analogous to viral RNA-dependent RNA polymerases in recognizing similar structures, when initiating de novo cDNAsynthesis. Thus, the variant plasmids incorporating tRNA and similar mtDNA sequences then integrate into the mitochondrial genome by homologous recombination. Given the high degree of sequence homology it can be surmised that the PMAD plasmids also act in a similar manner. It is still not clear how the plasmids became so patchily distributed in such geographically separated regions. The studies of Neurospora senescence made with the Maddur collection together with the findings of other researchers were reviewed by Maheshwari and Navaraj (2008).

\section{The possibility of confusing syntrophic growh with senescence}

A. Pandit, P. Delhi and Maheshwari showed that when conidia of two heterokaryon-incompatible auxotrophic $N$. crassa strains were mixed on minimal medium vigorous growth could sometimes occur via cross-feeding and without heterokaryon formation via hyphal fusion. The syntrophic growth often could be propagated through several subcultures using minute amounts of conidial inocula, but occasionally there was an unpredictable loss of growth potential in subcultures (Pandit et al., 1997). In cross-feeding, each strain supplements the growth factor required by the other without hyphal fusion. Loss of growth upon subculturing apparently resulted from 
the progressive loss of a particular conidial type from the disproportionate mixture in the small inocula. Phenotypically, this appeared similar to the senescence seen in some field isolates and for which no explanation had been found. Pandit et al., (1997) suggested that some of their wild-isolated Neurospora might indeed include mixtures of cross-feeding mutants that become unbalanced during conidial transfers.

\section{Perspectives for future research}

In the minireview, Maheshwari and Navaraj (2008) wrote: "It is expected that with the availability of the genome sequence of $N$. crassa, efforts will be made to identify ND and SEN". It has been more than ten years since this prediction, and as far as I am aware, the nd (natural death) and sen (senescent) genes have not yet been identified. The $\mathrm{mcb}$ and $\mathrm{mcm}$ genes also have not been identified. Nevertheless the field has moved on, researchers elsewhere have isolated additional nuclear genes whose mutation confers a senescent phenotype (Kothe et al., 2010; Yoshihara et al., 2017). That a lead developed by someone in India soon runs out of steam, but moves forward elsewhere is not uncommon (Kasbekar, 2016), and perhaps need not be lamented since scientific research is transnational. Fortunately, the mutants continue to be maintained by the Fungal Genetics Stock Center at Kansas State University, USA. Butcan they be maintained in perpetuity, especially given the precarious state of the FGSC's resources? It would be very unfortunate if the FGSC mutant collection was lost. Not only would one miss out on the thrill of using archived material to make discoveries in basic research, one would also lose the possibility of combating pathogenic fungi via molecular targeting of their ND or SEN homologues. There is a pressing need to identify the genes while the mutants are still available to be mapped. But who will do the mapping? A paralytic stroke cruelly cut short Maheshwari's research career just as the tools to map mutations to the nucleotide level had become available. My laboratory mapped and identified the fmf-1 mutant thirty years after it was found to uniquely lack the capacity to cross as either a male or female parent (lyer et al., 2009), but our motivation was to use the fmf-1 gene to implement a strategy to enrich for RIP-defective mutants (Box 1). Although our work gave us the "peace that passeth understanding" of the fmf-1 mutant phenotype, it fell short of our requirements for RIPdefective mutant enrichment. The ndand senmutants, belying their names, must be preserved for future generations of Neurospora researchers to work on.

\section{About Ramesh Maheshwari (adapted from a write-up prepared by Prof. Dipankar Nandi)}

Ramesh Maheshwari (19 December 1940 - 30 March 2019) was the son of Panchanan and Shanti Maheshwari. Panchanan Maheshwari was a well known botanist whose discovery of fertilization of angiosperms in a test tube led to the developments of new hybrid plants. Ramesh Maheshwari's brother is Satish Chandra Maheshwari, Professor at the University of Delhi who contributed in the area of plant physiology and molecular biology. Ramesh Maheshwari was awarded the PhD from the University of Wisconsin in 1966 for his research on rust fungi. He joined the Indian Institute of Science as a faculty on 4 April 1972, and retired on 31 July 2002. In addition to the studies described here, Maheshwari made significant contributions on thermophilic fungi, and one of his publications in this area has been cited more than 680 times.

Maheshwari authored the book "Fungi: experimental methods in Biology, "published by CRC press. Some time before his retirement, he suffered a stroke for which he had to be hospitalized. Due to a hospital borne infection, one of his legs was damaged and he would never be able to walk again. Remarkably, he recovered his health and post-retirement Maheshwari wrote interesting pedagogical articles for the journals Resonance, Journal of Biosciences, and Current Science. Colleagues recall him as a devoted teacher, and passionate scientist with an enormous commitment to the academic way of life (Adhvaryu, 2019).

\section{Acknowledgements}

Ramesh and Manjuli Maheshwari lavished warm hospitality on my earlier frequent visits to Bangalore, and their Malleswaram home, despite their gradually declining health. I thank Vidyanand Nanjundiah, K. Muniyappa, and N. B. Raju for suggestions to improve the article, and Vijay Mahajan for coining the phrase "update my ignorance" used vis-à-vis my inexperience with microconidia. I am an INSA Senior Scientist in CDFD, and an Honorary Visiting Scientist at CCMB.

\section{References}

ADHVARYU, K., (2019). Excellence by simplicity: Life and contributions of Prof. Ramesh Maheshwari (1940-2019). Fungal Genet. Rep. 63: Article 1.

BERTRAND, H., B. S.-S.CHAN and A. J. F. GRIFFITHS (1985). Insertion of a foreign nucleotide sequence into mitochondrial DNA causes senescence in Neurospora intermedia. Cell 41: 877-884.

BERTRAND, H., Q. WU and B. L. SEIDEL-ROGOL, (1993). Hyperactive recombination in the mitochondrial DNA of the natural death nuclear mutant of Neurospora crassa. Mol. Cell. Biol. 13: 6778-6788.

CHIANG, C. C., J. C. KENNELL, L. A. WANNER and A. M. LAMBOWITZ (1994).A mitochondrial retroplasmid integrates into mitochondrial DNA by a novel mechanism involving the synthesis of a hybrid cDNA and homologous recombination. Mol. Cell Biol. 14: 419-6432.

DODGE, B. O. (1936). Spermatia and nuclear migrations in Pleurage anserina Mycologia 28: 284-291.

D'SOUZA, A. D., H. BERTRAND and R. MAHESHWARI, (2005).Intramolecular recombination and deletions in mitochondrial DNA of senescent, a nuclear-gene mutant of Neurospora crassa exhibiting "death" phenotype. Fungal Genet. Biol 42: 178-190.

D'SOUZA, A. D., S. SULTANA and R. MAHESHWARI, (2005).Characterization and prevalence of a circular mitochondrial plasmid in senescence-prone isolates of Neurospora intermedia. Curr. Genet. 47: 182-193.

HE S. and N. E. SHARPLESS (2017).Senescence in health and disease. Cell 169 1000-1011.

IRELAN, J. T. and SELKER, E. U. (1996). Gene silencing in filamentous fungi: RIP, MIP and quelling. J. Genet. 75: 313-324.

IYER, S. V., M. RAMAKRISHNAN and D. P. KASBEKAR (2009). Neurospora crassa fmf-1 encodes the homologue of the Schizosaccharomyces pombe Ste11p regulator of sexual development. J. Genet. 88: 33-39.

KASBEKAR, D. P. (2016). History and development of genetics research in India: Three case studies. Ind. J. Hist. Sci. 51.2.2: 423-430.

KATO, A., K. KURASHIMA, M. CHAE, S SAWADA, S. HATAKEYAMA, S. TANAKA and $H$. INOUE (2010). Deletion of a novel F-box protein, MUS-10, in Neurospora crassa leads to altered mitochondrial morphology, instability of mtDNA and senescence. Genetics 185: 1257-1269.

KOTHE, G. O., M. KITAMURA, M. MASUTANI, E. U. SELKER and H. INOUE, (2010). PARP is involved in replicative aging in Neurospora crassa. Fungal Genet. Biol. 47: 297-309.

MAHESHWARI, R. (1989).Asexual reproduction without a mycelia phase in Neurospora. Fungal Genet. Newslett. 36: 48. 


\section{D.P. Kasbekar}

MAHESHWARI, R. (1991).Microcycle conidiation and its genetic basis in Neurospora crassa. J. Gen. Microbiol. 137: 2103-2115.

MAHESHWARI, R. (1999).Microconidia of Neurospora crassa. Fungal Genet. Biol. 26: 1-18.

MAHESHWARI R and A. ANTONY, (1974).A selective technique for the isolation of Neurospora crassa from soil. J. Gen. Microbiol. 81: 505-507.

MAHESHWARI R and A. NAVARAJ, (2008).Senescence in fungi: the view from Neurospora. FEMS Microbiol Lett. 280: 135-143.

MAHESHWARI R, A. PANDIT and B. KANNAN (1994).Senescence in strains of Neurospora from southern India. Fungal Genet Newslett. 41: 60.

NAVARAJ, A., A. PANDIT and R. MAHESHWARI (2000). senescent: a new Neurospora crassa nuclear gene mutant derived from nature exhibits mitochondrial abnormalities and a "death" phenotype. Fungal Genet. Biol. 29, 165-173.

PALANIVELU P and R. MAHESHWARI, (1979).Wild Neurospora isolated from soil. Neurospora Newsl. 26: 15.
PANDIT, A. and R. MAHESHWARI (1993).A simple method of obtaining pure microconidia in Neurospora crassa. Fungal Genet. Newslett. 40: 64-65.

PANDIT, A. and R. MAHESHWARI (1996).Life-history of Neurospora intermedia in a sugar cane field. J. Biosci. 21: 57-79.

PANDIT, A., P. DELHI and R. MAHESHWARI (1997).Syntrophic growth of auxotrophic strains of Neurospora crassa by cross-feeding. Fungal Genet. Newslett. 44:37-40.

SEIDEL-ROGOL, B. L., J. KING and H. BERTRAND, (1989). Unstable mitochondrial DNA in naturaldeath nuclear mutants of Neurospora crassa. Mol. Cell Biol. 9: 4259-4264.

SURYANARAYANAN, T. S. and S. S RATHINAKUMAR, (1984).Germ tube differentiation in some demetiaceous hyphomycetes as influenced by some surfaces. Comp. Physiol. Ecol. 9: 118-120.

YOSHIHARAR, Z. LI, K. ISHIMORI, K. KUWABARA, S. HATAKEYAMAand S. TANAKA (2017).Phenotypic analysis of newly isolated short-lifespan Neurospora crassa mutant deficient in a high mobility group box protein. Fungal Genet. Biol. 105:28-36. 


\section{Further Related Reading, published previously in the Int. J. Dev. Biol.}

Small molecules and cell differentiation in Dictyostelium discoideum

Tsuyoshi Araki and Tamao Saito

Int. J. Dev. Biol. (2019) 63: 429-438

Higher plant transformation: principles and molecular tools

Sylvester Anami, Elizabeth Njuguna, Griet Coussens, StijnAesaert and Mieke Van Lijsebettens

Int. J. Dev. Biol. (2013) 57: 483-494

https://doi.org/10.1387/ijdb.130232mv

Loss and gain of domains during evolution of cut superclass homeobox genes.

Thomas R Bürglin and Giuseppe Cassata

Int. J. Dev. Biol. (2002) 46: 115-123

Light-driven diurnal zonation in the filamentous fungus Fusarium solani.

$\mathrm{J}$ Das and $\mathrm{H}$ G Busse

Int. J. Dev. Biol. (1990) 34: 319-322

Development of progestin-specific response in the chicken oviduct.

P Tuohimaa, T Joensuu, J Isola, R Keinänen, T Kunnas, A Niemelä, A Pekki, M Wallén, T Ylikomi and M Kulomaa

Int. J. Dev. Biol. (1989) 33: 125-134
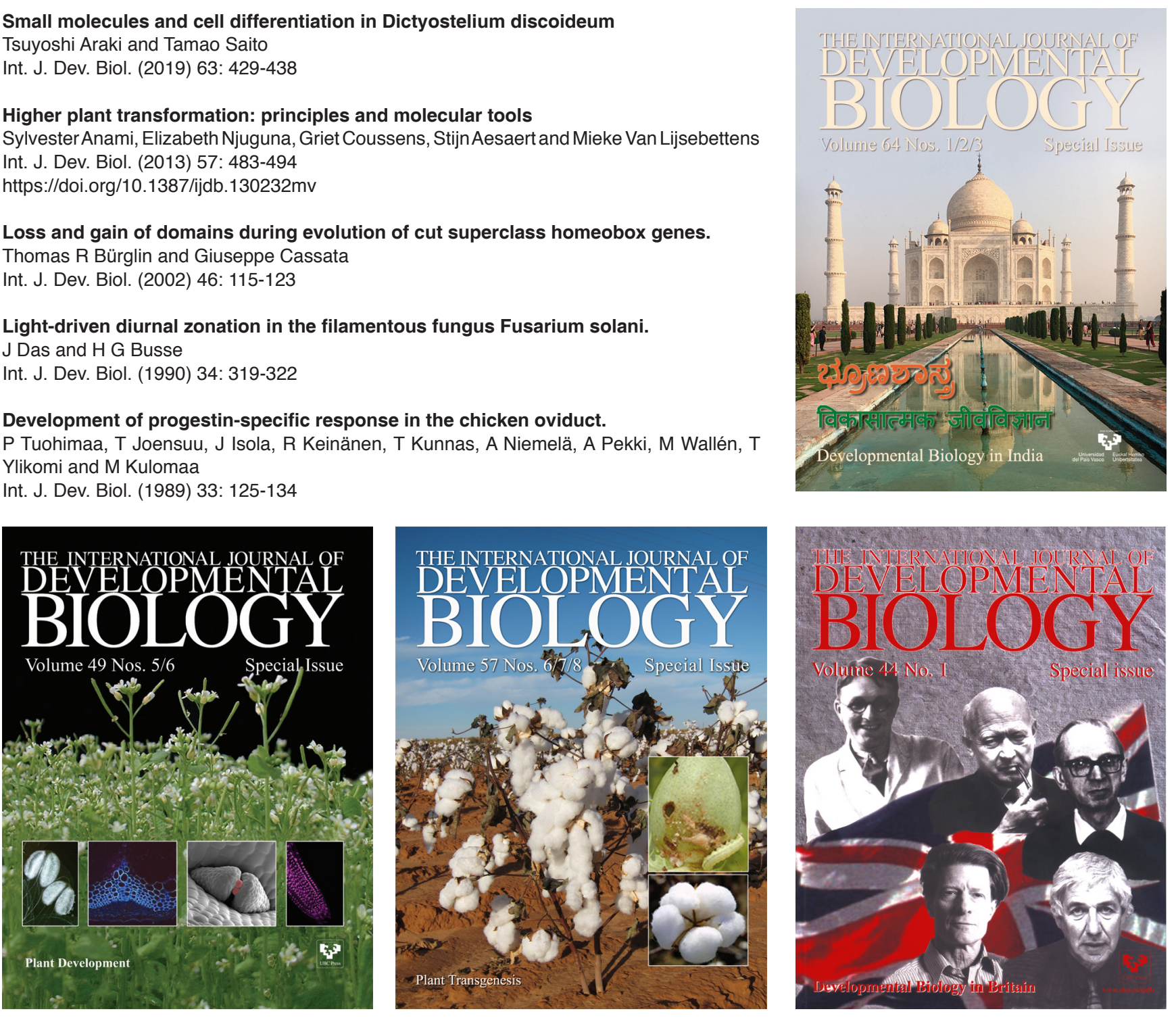